\title{
Century of manual remote control, automation, autonomy, and self-organization
}

\author{
Aarne Mämmelä \\ VTT Technical Research Centre \\ of Finland Ltd \\ Oulu, Finland \\ Aarne.Mammela@vtt.fi
}

\author{
Jukka Riekki \\ University of Oulu \\ Oulu, Finland \\ Jukka.Riekki@oulu.fi
}

\begin{abstract}
We present a multidisciplinary historical review of manual remote control, automation, autonomy, and selforganization roughly covering the last century. Some conceptual analysis is given using hierarchical classifications. We show the relationships between control theory, computer science, and communication theory. We observe that the three disciplines have progressed at least partially independently, but we can see also some convergence towards similar system models, often using different terminology. We expect that multidisciplinary studies will turn out to be useful for avoiding overlapping work and for making faster progress. Furthermore, a unified terminology would facilitate communication between disciplines. This review provides a starting point for building such terminology.
\end{abstract}

Keywords-remotely controlled systems; automatic systems; autonomous systems; self-organizing systems; multidisciplinary view

\section{INTRODUCTION}

We expect that future systems will be highly efficient which will need intelligent use of resources. By intelligence we mean the ability of a system to act appropriately in an uncertain environment to support the system's ultimate goal [1]-[3]. Uncertainty means lack of knowledge. Artificial intelligence is a theory of intelligent or rational agents. We observe that a goal is needed in all intelligent systems. It is a desirable state of the system in terms of performance or, in case of vehicles, a location. Usually high sophistication of intelligence implies high complexity that is measured by the use of basic resources. By basic resources we mean materials, energy, information (data and control), time (delay), frequency (bandwidth), and space (size) [4]. Information can be expressed in five levels, including statistics (including statistical properties), syntactics (format or mutual relationships), semantics (meaning or relationship to reality), pragmatics (meaning in a context), and apobetics (purpose) [5].

By efficiency we mean the ratio of benefits and expenditures where the benefits usually depend on the application (for example in communications they are data bits) and the expenditures are different basic resources. We are approaching fundamental limits in many areas and the limits form optimization constraints [4], [6]. In practice there are many expenditures, which leads to the use of constrained multiobjective optimization (MOO) and multiple-criteria decision making (MCDM) [7], [8] where the objectives or criteria are the different efficiency metrics, including for example throughput (in bit/s), delay (in ms), and reliability (in $\%$ ) [9]-[13]. If the throughput is normalized with bandwidth, power, and area, we obtain spectral efficiency (in bit/s/Hz), energy efficiency (in bit/s/W = bit/J), and area efficiency (in $\mathrm{bit} / \mathrm{s} / \mathrm{m}^{2}$ ), respectively. Regarding delay, a recent concept is age of information that is defined as the time interval from the birth of the information to the present time [14].

In this paper our focus is in the four basic system models: manual remote control, automation, autonomy, and selforganization. Their development is briefly explained during the last hundred years using a multidisciplinary approach [15]-[17] including control theory, computer science, and communication theory. Their hierarchical relationships are explained. The multidisciplinary view as an additive view is only the first step towards a holistic top-down systems view, which is the opposite of reductive analytical bottom-up view. Interdisciplinary view is a more advanced, interactive view and the third and most advanced, transdisciplinary view is the holistic systems view. Multidisciplinary system studies benefit from general dictionaries such as [18], [19]. Unified terminology is needed to facilitate personal communication between disciplines. Unfortunately such terminology is not yet complete.

This paper is a summary of a paper with wider scope [20]. The organization of the present paper is as follows. In Section II we present a three-level control hierarchy and a general hierarchy of systems. The four basic system models are described in Section III, including a historical perspective using the three disciplines. Section IV concludes the paper.

\section{HIERARCHICAL CONTROL}

Systems can in general be described by using functional, behavioral, structural, and physical levels from top down [15], [20]. Many systems only change the system behavior and leave the structure or organization untouched. The control or decision loop used for optimization is presented in Fig. 1 [21]. The loop includes three basic blocks that are sense, decide, and act blocks. The sense block receives state information from the process or plant. The information may be noisy. The decision block may receive control information from higher level decision blocks and send sensing information to the higher level decision blocks. The act block makes changes to the behavior or structure of the process. The control loop is used with different terminology in different disciplines, including learning loop in experiential learning; sense-plan-act paradigm in robotic systems; observe, 
orient, decide, and act loop (OODA) in combat operations process; cognitive cycle in cognitive radios; and monitor, analyze, plan, execute, and knowledge loop (MAPE-K) in autonomic computing.

The general three-level control hierarchy is presented in Fig. 2. The levels in the hierarchy are controller, coordinator, and organizer, which is also called manager. The number of levels can be smaller or larger. The terms differ in different disciplines. In communications, an orchestrator is sometimes used above the manager. The hierarchy was originally developed by Mesarovic (1970) and refined by Saridis (1977) [22], [23]. What we say below about the resolution and extent in time is valid also for the resolution and extent in frequency and space. In the frequency domain the extent is called the bandwidth.

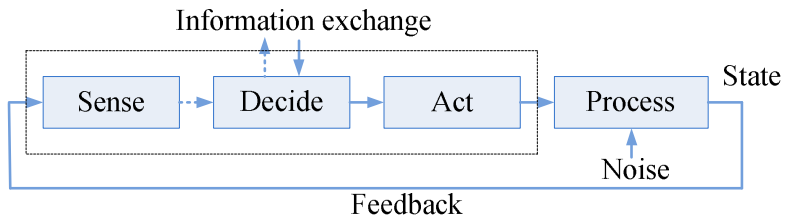

Fig. 1. Control loop.

\begin{tabular}{|c|}
\hline Hierarchy level \\
\hline $\begin{array}{c}\text { Organizer } \\
\text { (Manager) }\end{array}$ \\
\hline Coordinator \\
\hline Controller \\
\hline Process \\
\hline
\end{tabular}

Fig. 2. Control hierarchy.

The controller has the highest resolution in time and it has no memory and therefore it is not able to learn from past experience. The algorithms may be control or adaptive algorithms. The controller has high speed and accuracy, and therefore its complexity must be low, implying that its intelligence cannot be very high. The resolution is reduced and the extent is increased by at least one order of magnitude when moving one level upwards in the hierarchy. In such a case the hierarchy can be shown to minimize the entropy and the system is optimal in that sense [23].

The coordinator is the second level in the hierarchy. It has a short-term memory and thus it is able to learn. The algorithms are learning algorithms. The organizer or manager has a longterm memory. It has low resolution, speed, and accuracy, but large time extent. The organizer is able to do planning, which is a reasoning process by which a system predicts the future and selects the best course of action to achieve the goal [2]. The algorithms are self-organizing algorithms. The intelligence and therefore complexity may be high since the speed is slow.
In robotics the corresponding levels are behavioral, executive, and task-planning layers, respectively, but the names of the layers have not been unified [24], [25]. Robotics is heavily using results from control theory and computer science. The behavioral layer is responsible for movements and collision avoidance. The executive layer is responsible for choosing the current movement to achieve a task. The task-planning layer defines the long-term goals taking into account limited resources.

In communications the relevant levels in the Open Systems Interconnection (OSI) model (1984) are physical, data link, and network layer, respectively [26]. The OSI model was a joint effort of computer and communication scientists. The idea of the hierarchy is from control theory. In the physical layer bits or symbols are transmitted and the algorithms are control or adaptive algorithms, for example automatic frequency control or adaptive equalization and estimation. In the data link layer frames consisting of symbols are transmitted. For example, medium access control is used to coordinate the use of the link resources using a frame structure. In the network layer packets or datagrams are transmitted using the frames. The network layer is responsible for routing, which is an example of selforganization. In the physical layer the time resolution in present cellular systems is $32.5 \mathrm{~ns}$, in the data link layer it is $1 \mathrm{~ms}$ and in the network layer in the order of 1-10 s [27]. Thus there is several orders of magnitude difference, which enables increasing sophistication of algorithms at higher levels.

\begin{tabular}{|c|}
\hline System models \\
\hline Self-organizing systems \\
\hline Learning systems \\
\hline Adaptive systems \\
\hline Control systems \\
\hline Simple dynamic systems \\
\hline Static systems \\
\hline
\end{tabular}

Fig. 3. General hierarchy of systems.

The general hierarchy of systems is presented in Fig. 3. It was originally developed by Kenneth Boulding (1956) for natural systems [16], but we have modified it to cover humanmade systems. The lowest level includes static systems where everything is fixed. An example is a passive filter. No energy is consumed for information processing. The second level includes simple dynamic systems that are able to do periodic predetermined changes as in a clock. The third level includes control systems that include manually controlled and automatic systems using a feedback loop. Automatic systems use a setpoint value, which acts as a goal [21]. An example is proportional-integral-derivative (PID) control. The third level includes adaptive systems. They have a random reference signal replacing the set-point value, a performance criterion, and an algorithm [28], [29]. The performance criterion may be for example mean-square error (MSE) between the reference signal and system output. A common algorithm is the least-mean 


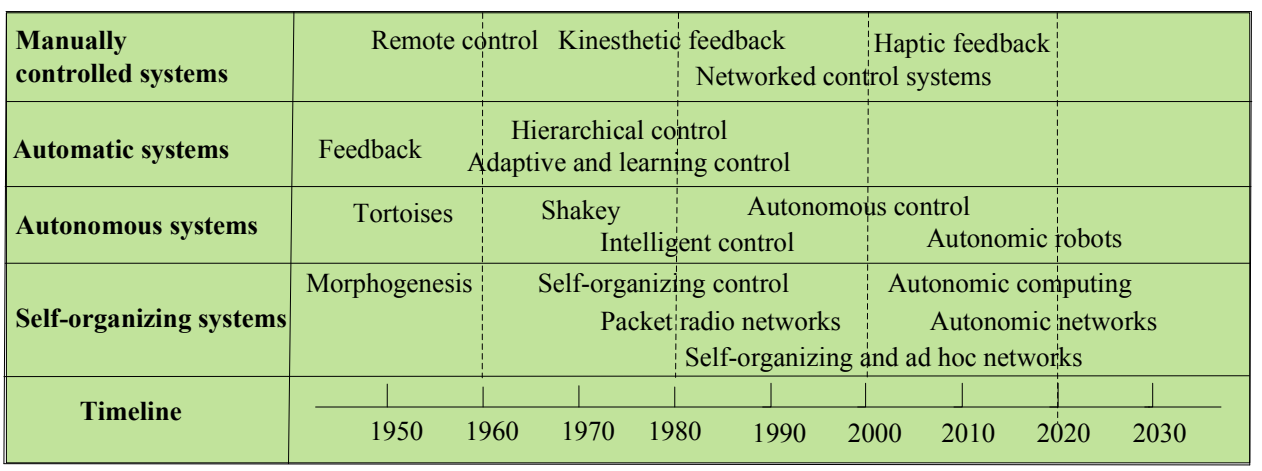

Fig. 4. Chronology of four system models. square (LMS) algorithm that can be used for parameter identification in a noisy environment where the changes are slow [30]. The LMS algorithm was devised by B. Widrow and R. E. Hoff in 1959, but its original versions were presented by R. von Mises and H. Pollaczek-Geiringer (1929) and Herbert Robbins and Sutton Monro (1951) [31]. The fifth level includes learning systems, which can modify their behavior using past experience in their memory [32]. They are sometimes called cognitive systems. The algorithms are machine learning algorithms that are based on supervised learning using a reference signal as in adaptive systems, unsupervised learning such as in pattern recognition, reinforcement learning such as in Q-learning, or evolutionary learning such as in genetic algorithms [34]. Autonomous and intelligent systems are advanced learning systems.

The uppermost level includes self-organizing systems which are autonomously able to change their structure. Examples include cooperating robots forming a society and self-organizing networks. In the self-organizing systems the algorithms are in general based on MOO and MCDM [7], [8]. The problems typically lack a unique solution but a trade-off between the objectives must be made. Hence, the solution has to be determined based on subjective preferences of the decision maker, that is, the decision makers select one of the reasonable alternatives they prefer. Fairness must be considered as well. Pareto optimal solutions are those solutions where no improvement in any objective can be made without worsening some other objective. The set of reasonable alternatives is typically identified using scalarization algorithms (e.g., weighted sum or weighted product algorithm). This works well if the multiple objectives are somewhat independent and monotonic, and the set of all possible solutions is convex. If not, random search or grid search can be used to find the optimum since otherwise a feedback algorithm may not find the global optimum, only a local one. Alternatively heuristic methods, metaheuristic algorithms (e.g., evolutionary algorithms, genetic algorithms, swarm intelligence, neural networks), and other optimization algorithms (fuzzy logic, game theory) can be used. Heuristic approaches differ from MOO since they are not guaranteed to find the optimal solution but they often find an approximate solution which is good enough.

\section{FOUR BASIC SYSTEM MODELS}

The four basic system models - manually controlled, automatic, autonomous, and self-organizing systems - differ in the sophistication of the control loop (Fig. 1). A chronology of these models is shown in Fig. 4. All of the systems have a long history in different disciplines, but finally they have converged to similar models with a somewhat different terminology.

\section{A. Manually and remotely controlled systems}

Manually controlled systems are the oldest of these system models. A human being in the control loop replaces the decision block (Fig. 1). The sense block is replaced by the human sensors (e.g., eyes, ears, and skin) and the act block with human actuators (e.g., hands, legs, and vocal tract) [21]. Most vehicles are still manually controlled since the required reaction times are short and complicated decisions must be made. Our ears have a reaction time of 140-160 ms including the delay of the brain, our eyes have a reaction time of 180-200 $\mathrm{ms}$, and the sense of touch has a reaction time of $155 \mathrm{~ms}$ [35]. A major fraction of the reaction time is used by the decision in the brain. The sense of touch can differentiate between two stimuli just $5 \mathrm{~ms}$ apart [36], [37]. Our ear can resolve clicks separated by $0.01 \mathrm{~ms}$ while the eye requires $25 \mathrm{~ms}$. Touch is highly sensitive to vibrations up to $1 \mathrm{kHz}$ with the peak sensitivity at about $250 \mathrm{~Hz}$.

A landmark book on remotely controlled systems is [38]. A modern term is networked control systems, which refers to control over a network [39]-[44]. One of the first networked control systems was the Control Area Network (CAN) developed in 1983-1986. Networked control systems and all other systems can be implemented as cyber-physical systems (2006), which are integrations of computation and physical processes, whose precursors were embedded systems [45]. Network induced constraints include time delays, packet losses and disorder, time varying transmission and sampling intervals, competition of multiple nodes accessing network, data quantization, clock asynchronization among local and remote nodes, and network security and safety [43].

The terms delay and latency are used synonymously. When defining a delay in a network, we must define the OSI layer, the protocol data unit (PDU), and whether it is a one-way or roundtrip delay. In practice, the delay refers to the total delay of a protocol data unit (PDU), which is a bit or symbol in the physical layer, a frame in the data link layer, a packet or datagram in the network layer, a segment in the transport layer, and message or bit in the application layer [26]. The delay includes processing, packetization, transmission, queuing, and propagation delays. Processing delays are caused by inefficient processing, for example in interleaving and automatic repeat request. Packetization delay is incurred in filling up a packet with data symbols. Transmission delay is caused by serial transmission. It is the delay between the transmission of the first and the last bit of a PDU. Queuing delays occur when buffers in network devices become flooded. Propagation delay is caused by the physical medium because of the finite propagation speed of the electromagnetic waves. Delays can be reduced by affecting each 
of these factors and limiting the physical size of the network. We must specify separately the data plane and control plane delays. Delay variation must be also taken into account [46].

The first remotely controlled system or telemanipulator was Nikola Tesla's wireless-controlled miniature boat (1898) [47]. The next tests were those by Raymond C. Goertz since about 1945 [38]. The results were later published with William M. Thompson in 1954. The operator manipulated radioactive materials. Audio-visual information was used in these systems. The first tests over the Internet were done by Ken Goldberg et al. (1995) [40]. Other senses have become recently important, especially the use of haptic information, which includes kinesthetic (sense of movement) and tactile (sense of touch) information. The first tests with kinesthetic feedback were made by William R. Ferrell (1966). Stability problems were observed with a delay of $100 \mathrm{~ms}$. The first review paper on haptic feedback was published in 1984, and the literature started to increase in 1991. A review paper on haptic Internet was published by Imad Elhajj et al. (2001) [48], [49]. Haptic interaction has been widely described in the literature [36], [37]. Later a similar concept based on tactile feedback was presented in [50]. All human senses are included in [51].

Using the early ideas on remote control, Minsky (1980) proposed a more general concept on telepresence instead of actual presence. In telepresence all the senses come into use, and the delays are minimized so that the interactions with the environment look almost instantaneous and an operator feels physically present at the remote site [38]. The three senses of sight, hearing, and touch are the most important. The delay across the Internet can be on the order of $100 \mathrm{~ms}$ or even more depending on the physical distance. The $100 \mathrm{~ms}$ requirement is the typical human reaction time below which the delay in human interactions with computers is not noticeable. The actual delays in present communication networks are $50-300 \mathrm{~ms}$ in the data link layer [27].

The delay requirement depends on the control task, but typically the tactile feedback delay should be between 5 and 50 ms [52]-[54]. The most demanding tasks in this respect are dragging on a touch screen (maximum delay of $1 \mathrm{~ms}$ ) and inking or line drawing using a stylus (maximum delay of $7 \mathrm{~ms}$ ). If the delay is too large, a human being may feel motion sickness called cybersickness. Low reliability causes the impression of time delay [44]. If the delay is limited to $1 \mathrm{~ms}$, the maximum radius of the network can be a few kilometers [55].

\section{B. Automatic systems}

Automatic systems are systems that do not need human intervention during their operation. Thus they are not manually controlled, and there is no human being in the control loop. They may still need some control signals from outside. If they are vehicles, the goal and the route to the goal are given to them before operation. If there is an obstacle in the route, an automatic system must stop to avoid collision and wait until the obstacle is removed. A landmark book on automatic systems is [21], but it does not include any advanced concepts such as hierarchy, degree of centralization, or adaptive, learning, and selforganizing control [22], [23], [29], [34], [56]. Hierarchy can be classified into nested (also called stratified), layered (multilayer), and dominance (multiechelon, organizational) hierarchy [22]. According to the degree of centralization, control systems can be divided into centralized and decentralized control, and their mixture called distributed control. For a history of control systems, see [47], [57]-[59],

Clocks are simple automata since they do not need manual intervention. However, usually automatic systems are based on the feedback concept using closed-loop control [21]. The optimum can be found iteratively or directly in one shot. Delays in a control loop cause instability and slowness of convergence. Sometimes a more rudimentary open-loop control is used since it may be faster although less accurate. In open-loop control no sensing information is used. The feedback was applied first in a water clock by Ktesibios in the third century BCE. Badi al-Jazari designed a humanoid automaton in the 13th century [25]. Leonardo da Vinci studied human anatomy and proposed a robot in about 1595, and in Japan mechanical dolls called Karakuri ningyo date back to at least the 18th century. A thermostat was invented in the beginning of the 1600's by Cornelis Dreppel [57]. James Watt used feedback in his steam engine (1769), and James Clark Maxwell developed the first mathematical analysis (1868). However, the analysis was forgotten for the next 80 years. Minorsky analyzed the working of human helmsman and developed the PID control system (1922) [21].

Harold S. Black invented the feedback amplifier (1927) but his patent was accepted only in 1937 since the concept was controversial at that time. His paper was published in 1934. The real start of modern automation was Harry Nyquist's stability analysis (1932). Nyquist was Black's assistant. After the analysis was published, the generality of the feedback concept was finally understood. Wiener (1948) combined control and communication theory in his cybernetics. The last century can be called the century or automation. The term became popular after Henry Ford opened his automation department (1947). Adaptive systems are advanced automatic systems. W. Ross Ashby developed the first adaptive system called homeostat as a simulation of the brain in 1948. The term adaptive control was first introduced in [60] by borrowing the term from biology. The first books on adaptive systems were published by Y. Z. Tsypkin [31] (originally published in Russian in 1968) and J. M. Mender and K. S. Fu [32]. The history is covered in [29].

\section{Autonomous systems}

Autonomous systems are automatic learning systems that do not need any external control signals during operation except the goal. Thus if the system is a vehicle, it would define its route to the goal by itself. If there is an obstacle, it is able to avoid collision by changing its route. Collision avoidance is thus an important feature in autonomous systems. A landmark book on such systems is [61].

The history of modern autonomous systems starts from W. Grey Walter's tortoises that were built in 1948-1949 [61]. In 1969 the next autonomous robot called Shakey was published by Nils J. Nilsson. The term autonomous control became more popular in 1989 after a review paper by Panos J. Antsaklis [62], [32]. Before that, autonomous systems were usually called intelligent systems, using the theories of artificial intelligence. The term intelligent control was introduced by King-Sun Fu 
(1971) [63], and further developed by George Saridis in his review paper [64]. Later some books on intelligent control have been published, for example, by James S. Albus, Alexander M. Meystel and George Saridis [2], [23], [65]. Autonomous systems are based on competition. If they are cooperating, they are sometimes called autonomic systems [66].

\section{Self-organizing systems}

Self-organizing systems are autonomous systems that are able to change their structure while the other system models change only the behavior. The structure is more difficult to change since it is "deeper" in the system. Above self-organizing systems would be self-replicating systems. Self-organization has been widely studied in biology since the times of Darwin. The term is now morphogenesis, which is considered as the greatest open problem in biology. It was first studied by D. W. Thompson (1917), W. Ross Ashby (1947), and Alan Turing (1952). Self-organizing control was started by Mihajlo D. Mesarovic (1964) and his work was continued by George Saridis (1977) [56], [67]. A self-organizing system can be described as a system that changes its own structure in order to improve the performance in an uncertain environment. A history of self-organizing robots is presented in [68]. In communications, self-organizing networks were originally called packet radio networks by Robert E. Kahn et al. (1972) and later ad-hoc networks, which term became popular in about 1994 when an IEEE subcommittee adopted the term. Perhaps the first to use the term self-organization in networking were Dennis J. Baker and Anthony Ephremides (1981). In computer science self-organizing systems are called autonomic computing systems (2001) [69], [70]. Following this, in communications they are called autonomic networks [71].

\section{CONCLUSION}

We expect that intelligent use of resources will stay important. We are approaching the fundamental limits of nature that form constraints and make necessary to use multiobjective optimization and multiple-criteria decision making. We have presented four important system models including manually controlled, automatic, autonomous, and self-organizing systems. They will be used in the future to complement each other. Remote controlled systems are now called networked control systems. Automatic systems are open-loop or closedloop control systems that do not need any manual control during operation. They may need external control such as a route and a goal. Autonomous systems are advanced automatic learning systems that do not need any external control except the goal. Intelligent systems are advanced autonomous systems that have the ability to act appropriately in an uncertain environment to support the system's ultimate goal that is always given from outside. Self-organizing systems can change their own structure in order to improve their performance in an uncertain environment. It is expected that future systems will be hierarchical and a combination of (1) conventional digital computers using logical deduction and (2) analog neural networks based on pattern recognition, thus complementing each other [16], [72]. A long-standing problem has been to place information into a context, implying the need for semantic information theory.
Multidisciplinary studies will turn out to be useful for avoiding overlapping work and for making faster progress. However, a unified terminology would facilitate communication between disciplines. This review provides a starting point for building such terminology.

\section{ACKNOWLEDGMENT}

This work was partially carried out at VTT in a project called AFORM5 where remote-controlled and autonomous machines with small delays and high reliability are considered. Comments from Antti Anttonen, Jyrki Huusko, Adrian Kotelba, and Tapio Heikkilä are acknowledged.

\section{REFERENCES}

[1] J. S. Albus, "Outline for a theory of intelligence," IEEE Transactions on Systems, Man, and Cybernetics, vol. 21, pp. 473-509, May/June 1991.

[2] J. S. Albus and A. M. Meystel, Engineering of Mind: An Introduction to the Science of Intelligent Systems. New York: John Wiley \& Sons, 2001.

[3] S. Russell and P. Norvig, Artificial Intelligence: A Modern Approach, 3rd ed. Upper Saddle River, NJ: Prentice-Hall, 2010.

[4] A. Mämmelä and A. Anttonen, "Why will computing power need particular attention in future wireless devices?" IEEE Circuits and Systems Magazine, vol. 17, pp. 12-26, First Quarter 2017.

[5] W. Gitt, "Information: The third fundamental quantity," Siemens Review, vol. 56, part 6, pp. 2-7, Nov.-Dec. 1989

[6] I. L. Markov, "Limits on fundamental limits to computation," Nature, vol. 512, pp. 147-154, 14 Aug. 2014.

[7] R. T. Marler and J. S. Arora, "Survey of multi-objective optimization methods for engineering," Struct. Multidiscipl. Optim., vol. 26, no. 6, pp. 369-395, Apr. 2004.

[8] J. Figueira, S. Greco, and M. Ehrgott (Eds.), Multiple-Criteria Decision Analysis: State of the Art Surveys. Boston, MA: Springer, 2005.

[9] A. Avizienis et al., "Basic concepts and taxonomy of dependable and secure computing," IEEE Transactions on Dependable and Secure Computing, vol. 1, no. 1, pp. 11-33, Jan.-Mar. 2004.

[10] M. Al-Kuwaiti et al., "A comparative analysis of network dependability, fault-tolerance reliability, security, and survivability," IEEE Communications Surveys \& Tutorials, vol. 11, pp. 106-124, Second Quarter 2009.

[11] B. Soret et al., "Fundamental tradeoffs among reliability, latency and throughput in cellular networks," in Proc. GLOBECOM'14 Workshops.

[12] B. Briscoe et al., "Reducing Internet latency: A survey of techniques and their merits," IEEE Communications Surveys \& Tutorials, vol. 18, pp. 2149-2196, Third Quarter 2016.

[13] M. Lévesque and D. Tipper, "A survey of clock synchronization over packet-switched networks,” IEEE Communications Surveys \& Tutorials, vol. 18, no. 4, pp. 2926 - 2947, Fourth Quarter 2016.

[14] A. Kosta et al., "Age of information: A new concept, metric, and tool," Foundations and Trends in Networking, vol. 12, no. 3, pp. 162-259, 2017.

[15] V. Hubka and W. E. Eder, Theory of Technical Systems: A Total Concept Theory for Engineering Design. Berlin: Springer-Verlag, 1988

[16] P. Checkland, Systems Thinking, Systems Practice, new ed. Chichester, UK: John Wiley \& Sons, 1999

[17] B. C. K. Choi and A. W. P. Pak, "Multidisciplinarity, interdisciplinarity and transdisciplinarity in health research, services, education and policy: 1. Definitions, objectives, and evidence of effectiveness," Clin. Invest. Med., vol. 29, pp. 351-364, Dec. 2006.

[18] Software and Systems Engineering Vocabulary (SE VOCAB), IEEE Computer Society and ISO/IEC JTC 1/SC7, 2018. [Online]. Available: https://pascal.computer.org.

[19] IEEE Standards Dictionary, IEEE, 2018. [Online]. Available: http://ieeexplore.ieee.org. 
[20] A. Mämmelä, J. Riekki, A. Kotelba, and A. Anttonen, "Multidisciplinary and historical perspectives for developing intelligent and resourceefficient systems," IEEE Access, vol. 6, pp. 17464-17499, 2018.

[21] K. Ogata, Modern Control Engineering, 5th ed. Boston, MA: PrenticeHall, 2010.

[22] M. Mesarovic, D. Macko, and Y. Takahara, Theory of Hierarchical, Multilevel Systems. New York: Academic Press, 1970.

[23] G. N. Saridis, Hierarchically Intelligent Machines. Singapore: World Scientific Pub Co, 2001.

[24] L. Nocks, The Robot: The Life Story of a Technology. Westport, CT: Greenwood Press, 2007.

[25] B. Siciliano and O. Khatib (Eds.), Springer Handbook of Robotics, 2nd ed. Berlin: Springer, 2016.

[26] J. F. Kurose and K. D. Ross, Computer Networking: A Top-Down Approach Featuring the Internet, 6th ed. Boston, MA: Addison-Wesley, 2013.

[27] H. Holma and A. Toskala, LTE for UMTS: Evolution to the LTEAdvanced, 2nd ed. Chichester, West Sussex, UK: John Wiley \& Sons, 2011.

[28] I. D. Landau, R. Lozano, M. M'Saad, and A. Karimi, Adaptive Control: Algorithms, Analysis and Applications. London: Springer, 2011.

[29] S. Haykin, Adaptive Filter Theory, 5th ed. Englewood Cliffs, NJ: Prentice-Hall, 2013.

[30] P. Monsen, "Fading channel communications," IEEE Communications Magazine, vol. 18, no. 1, pp. 16 - 25, Jan. 1980.

[31] Y. Z. Tsypkin, Adaptation and Learning in Automatic Systems. New York: Academic Press, 1972.

[32] J. M. Mendel and K. S. Fu, Adaptive, Learning, and Pattern Recognition Systems: Theory and Applications. New York: Academic Press 1970.

[33] P. J. Antsaklis and K. M. Passino (Eds.), An Introduction to Intelligent and Autonomous Control. Norwell, MA: Kluwer Academic Publishers, 1993.

[34] S. Marsland, Machine Learning: An Algorithmic Perspective, 2nd ed. New York: Chapman \& Hall/CRC, 2015.

[35] R. J. Kosinski, “A literature review on reaction time," Technical Note, Clemson University, Clemson, SC, Sep. 2013.

[36] A. El Saddik, M. Orozzo, M. Eid, and J. Cha, Haptics Technologies: Bringing Touch to Multimedia. Berlin: Springer 2011.

[37] R. S. Dahiya and M. Valle, Robotic Tactile Sensing: Technologies and System. New York: Springer, 2013.

[38] T. B. Sheridan, Telerobotics, Automation, and Human Supervisory Control. Cambridge, MA: MIT Press, 1992.

[39] R. A. Gupta and M.-Y. Chow, "Networked control system: Overview and research trends," IEEE Transactions on Industrial Electronics, vol. 57, pp. 2527-2535, Jul. 2010

[40] P. F. Hokayem and M. W. Spong, "Bilateral teleoperation: An historical survey," Automatica, vol. 42, no. 12, pp. 2035-2057, 2006.

[41] W. Zhang et al., "Stability of networked control systems," IEEE Control Systems Magazine, vol. 21, pp. 84-99, Feb. 2001.

[42] G. C. Walsh and H. Ye, "Scheduling of networked control systems," IEEE Control Systems Magazine, vol. 21, pp. 57-65, February 2001.

[43] L. Zhang et al., "Network-induced constraints in networked control systems-A survey," IEEE Transactions on Industrial Informatics, vol. 9, pp. 403-416, Feb. 2013.

[44] Z. Shi et al., "Effects of packet loss and latency on temporal discrimination of visual-haptic events," IEEE Trans. Haptics, vol. 3, no. 1, pp. 28-36, Jan./Mar. 2009.

[45] K. D. Kim and P. R. Kumar, "Cyber-physical systems: A perspective at the centennial," Proc. IEEE, vol. 100, Special Centennial Issue, pp. 1287 - 1308, May 2012.

[46] ITU-T Y.1540, "Internet protocol aspects - Quality of service and network performance, Internet protocol data communication service - IP packet transfer and availability performance parameters," July 2016.
[47] S. Y. Nof (Ed.), Springer Handbook of Automation. Berlin: Springer, 2009.

[48] I. Elhajj et al., "Haptic information in Internet-based teleoperation," IEEE/ASME Transactions on Mechatronics, vol. 6, no. 3, pp. 295 - 304, Sep. 2001.

[49] A. Aijaz et al., "Realizing the tactile Internet: Haptic communication over next generation 5G cellular networks," IEEE Wireless Communications, vol. 24, no. 2, pp. 82-89, Apr. 2017.

[50] G. P. Fettweis, “The tactile Internet: Applications and challenges," IEEE Vehicular Technology Magazine, vol. 9, pp. 64-70, Mar. 2014.

[51] R. Prasad, "Human bond communication," Wireless Personal Communications, vol. 87, pp. 619-627, 2016.

[52] T. Kaaresoja et al., "Towards the temporally perfect virtual button: Touch-feedback simultaneity and perceived quality in mobile touchscreen press interactions," ACM Trans. Appl. Percept., vol. 11, no. 2, pp. 9:19:25, 2014.

[53] C. Attig et al., "System latency guidelines then and now - Is zero latency really considered necessary?" in Engineering Psychology and Cognitive Erogonomics: Cognition and Design, D. Harris, Ed. Cham, Switzerland: Springer, 2017, pp. 3-33.

[54] M. Annett, “(Digitally) Inking in the 21st century,” IEEE Computer Graphics and Applications, vol. 37, no. 1, pp. 92-99, Jan.-Feb. 2017.

[55] M. Simsek et al., "5G-enabled tactile Internet," IEEE J. Sel. Areas. Commun., vol. 34, no. 3, pp. 460-473, Mar. 2016.

[56] G. N. Saridis, Self-Organizing Control of Stochastic Systems. New York: Marcel Dekker 1977.

[57] O. Mayr, The Origins of Feedback Control. Stevenage, UK: Peter Peregrinus, 1970.

[58] S. Bennett, A History of Control Engineering, 1930-1955. Clinton, MA: Colonial Press, 1993.

[59] S. Bennett, A History of Control Engineering, 1800-1930. Clinton, MA: Colonial Press, 1986.

[60] R. F. Drenick and R. A. Shahbender, "Adaptive servomechanisms," Transactions of the American Institute of Electrical Engineers, Part II: Applications and Industry, vol. 76, pp. 286 - 292, Nov. 1957.

[61] G. A. Bekey, Autonomous Robots: From Biological Inspiration to Implementation and Control. Cambridge, MA: MIT Press, 2005.

[62] P. J. Antsaklis et al., "Towards intelligent autonomous control systems: Architecture and fundamental issues," Journal of Intelligent and Robotic Systems, vol. 1, pp. 315-342, 1989.

[63] K. S. Fu, "Learning control systems and intelligent control systems: An Intersection of artificial intelligence and automatic control," IEEE Transactions on Automatic Control, vol. AC-16, pp. 70-72, Feb. 1971.

[64] G. N. Saridis, "Toward the realization of intelligent controls," Proc. IEEE, vol. 67, pp. 1115-1133, Aug. 1979.

[65] A. M. Meystel and J. S. Albus, Intelligent Systems: Architecture, Design, and Control. New York: John Wiley \& Sons, 2002.

[66] M. Schaefer et al., "Multi-agent traffic simulation for development and validation of autonomous car-to-car systems" in Autonomic Road Transport Systems, T. L. McCluskey, A. Kotsialos, J. P. Muller, F. Klugl, O. Rana, and R. Schumann (Eds.), Springer International Publishing Switzerland, 2016, pp. 165-180.

[67] M. D. Mesarovic, "Self-organizing control systems," IEEE Transactions on Applications and Industry, vol. 83, no. 74, pp. 265-269, Sep. 1964.

[68] S. Murata and H. Kurokawa, Self-Organizing Robots. Springer, 2012.

[69] J. O. Kephart and D. M. Chess, "The vision of autonomic computing," Computer, vol. 36, no. 1, pp. 41 - 50, Jan. 2003.

[70] S. Dobson et al., "Fulfilling the vision of autonomic computing," Computer, vol. 43, pp. 35-41, Jan. 2010.

[71] S. Dobson et al., "A survey of autonomic communications," ACM Transactions on Autonomous and Adaptive Systems, vol. 1, no. 2, pp. 223-259, Dec. 2006

[72] T. Stonier, Information and Meaning: An Evolutionary Perspective. Berlin: Springer, 1997 